\title{
Fishing for Precision in ALK-Rearranged Non-Small-Cell Lung Cancers
}

Xiuning Le ${ }^{1}$, Mary Linton Peters ${ }^{1}$, Paul A VanderLaan², Daniel B Costa1 and Deepa Rangachari ${ }^{1 *}$

${ }^{1}$ Department of Medicine, Division of Hematology/Oncology, Beth Israel Deaconess Medical Center, Harvard Medical School, Boston, MA, USA

${ }^{2}$ Department of Pathology, Beth Israel Deaconess Medical Center, Harvard Medical School, Boston, MA, USA

\begin{abstract}
Fluorescence in situ hybridization (FISH) and immunohistochemistry (IHC) represent the current standard for identification of therapeutically relevant anaplastic lymphoma kinase (ALK) rearrangements in advanced non-small-cell lung cancers (NSCLCs). Use of tyrosine kinase inhibitors (TKIs) targeting the oncogenic ALK fusion protein is approved for use in advanced ALK-rearranged NSCLCs and with benefits both with regards to clinical outcomes and quality of life when compared with traditional palliative cytotoxic therapies. We report here 2 cases in which initial standard of care ALK testing by FISH yielded false positive results, leading to delays in determination of optimal systemic therapy.
\end{abstract}

Keywords: Non-small cell lung cancer; NSCLC; ALK; Crizotinib, FISH; Targeted therapy

\section{Introduction}

Roughly 3-7\% of non-small-cell lung cancers (NSCLCs) harbor anaplastic lymphoma kinase $(A L K)$ rearrangements that are responsive to the tyrosine kinase inhibitor (TKI) crizotinib. Since August 2011, the Vysis $A L K$ break apart FISH probe (Abbott Molecular, Inc.), had been the sole United States (US) Food and Drug Administration (FDA)approved method for detection of $A L K$ rearrangements in NSCLC, with the cutoff for $A L K$ positivity defined as $\geq 15 \%$ of cells. However, as of June 2015, the VENTANA ALK (D5F3) CDx IHC assay (Ventana Medical Systems, Inc.) has been additionally approved. False negative and discordant $A L K$ FISH results have been reported [1,2]. Here, we report patients who received false positive results on $A L K$ FISH, leading to delay of optimal systemic therapy. ALK FISH testing in both instances was performed as a send out test by a certified commercial vendor utilizing the FDA-approved companion assay (Vysis ALK Break Apart FISH Kit, Abbott Molecular).

\section{Case 1}

An 83 year-old gentleman with a prior $<10$ pack/years tobacco history presented with metastatic lung adenocarcinoma (LAC) with malignant pleural effusion (Figure 1A). Single gene assays were completed on formalin-fixed, paraffin-embedded (FFPE) cell blocks created from the pleural fluid cytologic specimen and showed $16 \%$ of cells with an $A L K$ rearrangement by FISH; EGFR, KRAS, and ROS1 were unaffected. The patient was considered for a clinical trial (NCT02075840); however, central confirmation of tumor $A L K$ status by IHC showed no evidence of an $A L K$ rearrangement (IHC score was 0). Crizotinib $250 \mathrm{mg}$ twice daily was started. Follow-up one month later demonstrated clinical and radiographic disease progression (Figure 1B). Right pleural biopsy and targeted next generation sequencing (SNaPshot NGS and fusion gene assay, Massachusetts General Hospital) demonstrated an EGFR exon 19 deletion, with no evidence of $A L K$ gene fusion. Crizotinib was discontinued, and erlotinib at a dose of $150 \mathrm{mg}$ daily was started. This resulted in a partial radiographic response with improvement in clinical symptoms that has been sustained for 6 months (Figure 1C).

\section{Case 2}

A 64 year-old woman with a prior 10 pack/years tobacco history presented with metastatic LAC with malignant pleural effusion (Figure 2A). Sequential single gene assays were completed on FFPE cell blocks created from the pleural fluid cytologic specimen. The tumor was found to harbor both $A L K$ rearrangement by FISH in $>90 \%$ of cells and
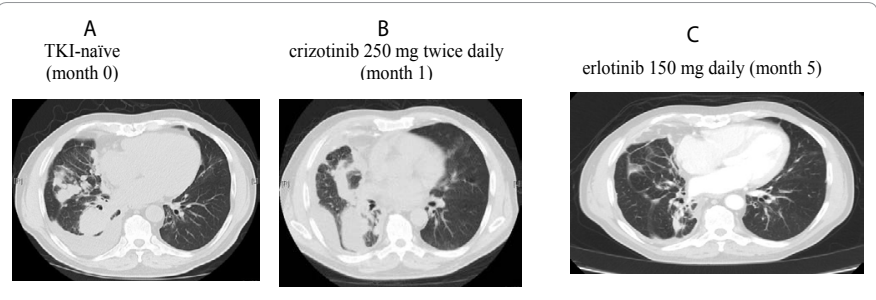

Figure 1: Computerized tomography (CT) images of the chest for patient 1 over the course of treatment. (A) At the time of diagnosis. (B) After one month of crizotinib therapy. C.) Five months after erlotinib therapy. Single gene assay (SGA) and next generation sequencing (NGS) results are displayed Patient no. 1, tumor SGA: EGFR wild-type, ALK FISH positive (nuc ish(ALKx2 3) (5'ALK sep 3'ALKx1)[7/100]/ (3'ALKx2,5'ALKx1)(3'ALK con 5'ALKx1)[4/100]/ (3'ALKx3,5'ALKx2)(3'ALK con 5'ALKx1)[5/100]) tumor NGS: EGFR-delE746_ $\mathrm{R} 748+\mathrm{A} 750 \mathrm{P}$, no $A L K$ rearrangement.

concurrent EGFR exon 19 mutation. Crizotinib $250 \mathrm{mg}$ twice daily was started. However, symptomatic and radiographic progression following six weeks of therapy was noted (Figure $2 \mathrm{~B}$ ). Repeat testing on the same cytologic specimen again showed an $A L K$ rearrangement by FISH; however, NGS showed the EGFR exon 19 mutation only. Crizotinib was discontinued, and erlotinib $150 \mathrm{mg}$ daily was started. Eight weeks later, imaging showed a partial response that has been sustained for more than 9 months (Figure 2C).

\section{Discussion}

With the dramatic responses and tolerability observed with the use of crizotinib in ALK-rearranged NSCLC, it has become imperative to perform timely and accurate molecular testing. Currently, both FISH and IHC serve as FDA-approved options for testing. Nevertheless, there have been an increasing number of reports highlighting false negative results using FISH, on the order of 5-15\% [3]. False negative results

*Corresponding author: Deepa Rangachari, Division of Hematology/Oncology, Beth Israel Deaconess Medical Center, 330 Brookline Avenue, Shapiro 9 Boston, MA 02215, United states, Tel: 617-667-1580; Fax: 617-667-2518; E-mail: drangach@bidmc.harvard.edu

Received: October 22, 2015; Accepted: December 03, 2015; Published: December 07, 2015

Citation: Le X, Peters ML, VanderLaan PA, Costa DB, Rangachari D (2015) Fishing for Precision in ALK-Rearranged Non-Small-Cell Lung Cancers. Oncol Cancer Case Rep 1:105.

Copyright: () 2015 Le X, et al.. This is an open-access article distributed under the terms of the Creative Commons Attribution License, which permits unrestricted use, distribution, and reproduction in any medium, provided the original author and source are credited. 


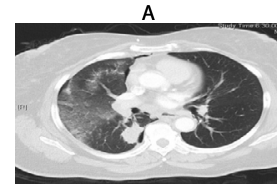

TKI-naïv (month 0)

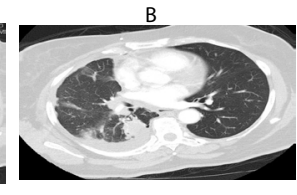

crizotinib $250 \mathrm{mg}$ twice daily (month 1.5)

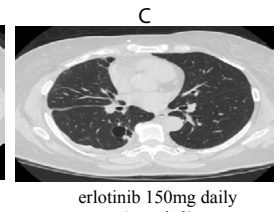

(month 8)
Figure 2: Computerized tomography (CT) images of the chest for patient 2 over the course of treatment. (A) At the time of diagnosis. (B) After six weeks of crizotinib therapy. C.) Eight months after erlotinib therapy. Single gene assay (SGA) and next generation sequencing (NGS) results are displayed. Patient no. 2: tumor SGA: EGFR-delE746 T751insl; ALK FISH positive (nuc ish(3'ALKx2 3,5'ALKx1 2)(3'ALK con 5'ALKx1 2)[46/50]) tumor NGS: EGFRdelE746_T751insl, no ALK rearrangement.

may occur due to variant forms of EML4-ALK rearrangements and/or RNA editing abnormalities resulting in intron changes that cannot be detected by FISH [4]. Several groups have published on the comparative accuracy of FISH, IHC, and NGS; however, the sensitivity, specificity, and cost of these modalities have not been directly compared in large prospective trials to date [3-6]. We report here two cases with clinically false positive FISH results. In both cases, initiation of crizotinib resulted in rapid disease progression and warranted further interrogation by NGS. Interestingly, both tumors were found to harbor EGFR mutations and with brisk and ongoing responses to erlotinib. The practice of precision medicine is incumbent upon use of assays which detect drug targets with optimal accuracy. As NGS becomes increasingly accessible and cost effective and as the spectrum of actionable therapeutic targets continues to expand, NGS may replace other modalities for optimally pairing patients with best therapies especially in cases where there is a paradoxical/unexpected response to therapy that is inconsistent with the predictive biomarker assayed.

\section{References}

1. Sholl LM WS, Gray SW, Wong KK, Chirieac LR, Lindeman NI, et al. (2013) Combined use of ALK immunohistochemistry and FISH for optimal detection of ALK-rearranged lung adenocarcinomas. Journal of thoracic oncology : official publication of the International Association for the Study of Lung Cancer 8: 322-328.

2. Peled N, Palmer G, Hirsch FR (2012) Next-generation sequencing identifies and immunohistochemistry confirms a novel crizotinib-sensitive ALK rearrangement in a patient with metastatic non-small-cell lung cancer. Journal of thoracic oncology : official publication of the International Association for the Study of Lung Cancer 7: e14-6.

3. Ali SM OS, He J, Peled N, Chmielecki J, Pinder MC, et al. (2014) Identifying ALK rearrangements that are not detected by FISH with targeted next-generation sequencing of lung carcinoma. J Clin Oncol 32: $5 \mathrm{~s}$.

4. Pekar-Zlotin M, Hirsch FR, Soussan-Gutman L (2015) Fluorescence in situ hybridization, immunohistochemistry, and next-generation sequencing for detection of EML4-ALK rearrangement in lung cancer. The oncologist 20: 316322.

5. Selinger $\mathrm{Cl}$, Rogers TM, Russell PA (2013) Testing for ALK rearrangement in lung adenocarcinoma: a multicenter comparison of immunohistochemistry and fluorescent in situ hybridization. Modern pathology: an official journal of the United States and Canadian Academy of Pathology 26: 1545-1553.

6. Minca EC, Portier BP, Wang Z (2013) ALK status testing in non-small cell lung carcinoma: correlation between ultrasensitive IHC and FISH. The Journal of molecular diagnostics : JMD 15: 341-346. 